\title{
Epigenetic Modulation by Apabetalone Counters Cytokine-Driven Acute Phase Response In Vitro, in Mice and in Patients with Cardiovascular Disease
}

\author{
Sylwia Wasiak, ${ }^{1}$ Dean Gilham, ${ }^{1}$ Emily Daze, ${ }^{1}$ Laura M. Tsujikawa, ${ }^{1}$ Christopher Halliday, ${ }^{1}$ \\ Stephanie C. Stotz, ${ }^{1}$ Brooke D. Rakai, ${ }^{1}$ Li Fu, ${ }^{1}$ Ravi Jahagirdar, ${ }^{1}$ Michael Sweeney, \\ Jan O. Johansson, ${ }^{2}$ Norman C. W. Wong, ${ }^{1}$ and Ewelina Kulikowski ${ }^{1}{ }^{1}$
}

${ }^{1}$ Resverlogix Corp., Calgary, AB, Canada

${ }^{2}$ Resverlogix Inc., San Francisco, CA, USA

Correspondence should be addressed to Ewelina Kulikowski; ewelina@resverlogix.com

Received 30 April 2020; Accepted 6 July 2020; Published 1 August 2020

Academic Editor: Leonardo De Luca

Copyright (C) 2020 Sylwia Wasiak et al. This is an open access article distributed under the Creative Commons Attribution License, which permits unrestricted use, distribution, and reproduction in any medium, provided the original work is properly cited.

\begin{abstract}
Chronic systemic inflammation contributes to cardiovascular disease (CVD) and correlates with the abundance of acute phase response (APR) proteins in the liver and plasma. Bromodomain and extraterminal (BET) proteins are epigenetic readers that regulate inflammatory gene transcription. We show that BET inhibition by the small molecule apabetalone reduces APR gene and protein expression in human hepatocytes, mouse models, and plasma from CVD patients. Steady-state expression of serum amyloid P, plasminogen activator inhibitor 1, and ceruloplasmin, APR proteins linked to CVD risk, is reduced by apabetalone in cultured hepatocytes and in humanized mouse liver. In cytokine-stimulated hepatocytes, apabetalone reduces the expression of C-reactive protein (CRP), alpha-2-macroglobulin, and serum amyloid P. The latter two are also reduced by apabetalone in the liver of endotoxemic mice. BET knockdown in vitro also counters cytokine-mediated induction of the CRP gene. Mechanistically, apabetalone reduces the cytokine-driven increase in BRD4 BET occupancy at the CRP promoter, confirming that transcription of CRP is BET-dependent. In patients with stable coronary disease, plasma APR proteins CRP, IL-1 receptor antagonist, and fibrinogen $\gamma$ decrease after apabetalone treatment versus placebo, resulting in a predicted downregulation of the APR pathway and cytokine targets. We conclude that CRP and components of the APR pathway are regulated by BET proteins and that apabetalone counters chronic cytokine signaling in patients.
\end{abstract}

\section{Introduction}

Liver-derived acute phase response (APR) proteins are a central component of the inflammatory response that is induced by pathogens, trauma, environmental stress, or sterile tissue injury [1]. In response to proinflammatory cytokines, hepatocytes downregulate the synthesis of several steady-state proteins (termed "negative" APR proteins) and upregulate the secretion of "positive" APR proteins [2]. IL-6 and IL-1 $\beta$ signal through transmembrane receptors on hepatocyte membrane to activate nuclear transcription factors that control APR gene transcription, including the signal transducer and activator of transcription 3 (STAT3) and the nuclear factor $\kappa \mathrm{B}(\mathrm{NF}-\kappa \mathrm{B})$ [2]. Costimulation with IL-6 and IL- $1 \beta$ leads to coordinated loading of NF- $\kappa \mathrm{B}$ and STAT3 onto gene enhancers marked by increased acetylation of histone 3 lysine 27 residues $(\mathrm{H} 3 \mathrm{~K} 27 \mathrm{ac})$, resulting in a synergistic induction of gene expression [3]. Histone acetylation marks are detected by members of the bromodomain and extraterminal (BET) protein family, presenting the possibility that BETs also play a role in APR gene transcription [4]. BET proteins bind to acetylated lysines through two short peptide motifs called bromodomains (BD) 1 and 2 [4]. The BET protein BRD4 also contains a unique $\mathrm{C}$-terminal domain that binds the positive transcription elongation factor $\mathrm{P}-\mathrm{TEFb}$, directly stimulating RNA polymerase II-dependent transcription [5, 6]. Notably, BRD4-dependent transcription is associated with the induction of transcriptional programs that drive pathogenic 
conditions including chronic inflammation [7, 8]. Whether $\mathrm{BRD} 4$ plays a direct role in regulating APR gene transcription during acute and chronic inflammation is currently unknown.

Apabetalone (RVX-208) is a small molecule inhibitor of BET proteins (BETi) that selectively targets the second $\mathrm{BD}$ (BD2) of BET proteins, reducing their transcriptional activity $[9,10]$. Preclinical and clinical studies have shown that apabetalone modulates the expression of proinflammatory and proatherosclerotic genes in models of atherosclerosis and in patients with coronary artery or chronic kidney disease [11-17]. Apabetalone also impacts the transcription of APR genes in primary human hepatocytes $(\mathrm{PHH})$, including C-reactive protein (CRP) $[12,13,16]$. Considering that CRP is a key biomarker of cardiovascular (CVD) risk assessment and treatment [18], it is important to understand its hepatic transcriptional regulation during acute and chronic inflammation. In this study, we use apabetalone in vitro and in vivo to further delineate the role of BET proteins in the transcriptional regulation of the APR pathway, with a particular focus on CRP. For the first time, we show that cytokine-mediated induction of CRP gene expression is BET-dependent. Further, we show that BRD4 occupancy on the CRP promoter increases following cytokine stimulation and that this association is inhibited by BETi. Finally, we provide evidence that apabetalone treatment of patients with cardiovascular disease (CVD) reduces the abundance of proteins within cytokine and APR pathways, potentially reducing the inflammatory risk associated with CVD.

\section{Methods}

2.1. Chemical Synthesis. Apabetalone and JQ1 were synthesized by NAEJA Pharmaceuticals (Edmonton, Canada) or IRIX Pharmaceuticals (Florence, SC) [12].

2.2. Tissue Culture. For gene expression and protein secretion studies, cryopreserved primary human hepatocytes from adult donors were plated as recommended (CellzDirect, Life Technologies). Cells were treated with compounds dissolved in DMSO (0.05-0.1\%) \pm cytokines $(10 \mathrm{ng} / \mathrm{mL})$ in media with $10 \%$ fetal bovine serum (FBS) $(v / v)$ but without dexamethasone (CellzDirect, Life Technologies) for up to $72 \mathrm{~h}$. For protein secretion studies, culture media were collected over the final $24 \mathrm{~h}$ of the experiment. HepaRG ${ }^{\mathrm{TM}}$ cells were plated as recommended (ThermoFisher Scientific). On day 6 postplating, cells were treated with cytokines \pm compounds for 2 or $24 \mathrm{~h}$. For short-term treatments, cells were preincubated with BET inhibitors for $1 \mathrm{~h}$ prior to addition of cytokines; treatments longer than $6 \mathrm{~h}$ received both agents simultaneously.

2.3. Quantification of RNA Expression. For gene expression studies, primary hepatocytes and HepaRG ${ }^{\mathrm{TM}}$ cells were harvested using the mRNA Catcher PLUS kit (ThermoFisher Scientific). Gene expression was quantified by RNA UltraSense $^{\mathrm{TM}}$ One-Step qRT-PCR System (ThermoFisher Scientific). Levels of messenger RNA (mRNA) of interest were measured by human TaqMan ${ }^{\mathrm{TM}}$ Gene Expression Assays (ThermoFisher Scientific) relative to the endogenous control cyclophilin A in a duplex reaction. Data were acquired using the ViiA-7 rtPCR System (ThermoFisher Scientific).

2.4. ELISA. To assess the APR protein secretion, culture media from primary human hepatocyte cultures were collected over the final $24 \mathrm{~h}$ of treatment, flash-frozen in liquid nitrogen, and stored at $-80^{\circ} \mathrm{C}$ prior to analysis. Secreted proteins were detected with AssayMax ${ }^{\mathrm{TM}}$ ELISA Kits (AssayPro).

2.5. Western Blots. Proteolysis targeting chimera (PROTAC) MZ-1 (Tocris) was added at $0.1-0.8 \mu \mathrm{M}$ for $24 \mathrm{~h}$ prior to cytokine treatment. HepaRG ${ }^{\mathrm{TM}}$ cell lysates were prepared in PBS $+2 \%$ SDS and sonicated $3 \times 10 s$ with a Branson SLPt sonicator (Branson Ultrasonics). Insoluble material was removed by centrifugation and protein content was assessed with the DC ${ }^{\mathrm{TM}}$ Protein Assay (Bio-Rad). Proteins were resolved by SDS-PAGE, transferred to nitrocellulose, and probed with antibodies against BRD2, BRD3, or BRD4 (Bethyl), followed by HRP-coupled secondary antibodies (Calbiochem). $\beta$-Actin was detected with antibodies coupled to HRP (Sigma-Aldrich). Luminescent signal was revealed by Amersham ECL Plus chemiluminescent reagent (GE Healthcare Life Sciences) and quantified with the Quantity One 1D analysis software (Bio-Rad).

2.6. Chromatin Immunoprecipitation. To assess BET protein chromatin occupancy, HepaRG ${ }^{\mathrm{TM}}$ cells were pretreated with BET inhibitors for 1 hour before addition of IL- 6 and IL- $1 \beta$ $(10 \mathrm{ng} / \mathrm{mL})$ for a $2 \mathrm{~h}$ coincubation period. Cells were crosslinked with formaldehyde, and Active Motif Inc. (Carlsbad, CA) performed chromatin isolation and immunoprecipitation with BRD2 or BRD4 antibodies (Bethyl). Samples were processed in triplicate. Statistical significance was determined through 2-way ANOVA followed by Tukey's multiple comparison test for within-group comparison or Sidak's test for between-group comparison. A $p$ value $\leq 0.05$ was considered statistically significant.

\subsection{Mouse Models}

2.7.1. Humanized Chimeric Mouse Model. Urokinase-type plasminogen activator (uPA)/severe combined immunodeficient (SCID) mice with livers repopulated with human hepatocytes were generated as described previously [13] (PhoenixBio, Co., Ltd., Higashihiroshima, Japan). Protocols for the animal experiments were approved by the Laboratory Animal Ethics Committee at PhoenixBio Co., Ltd. (Resolution No. 0740). In vivo experiments were performed in a facility approved by the Office of Laboratory Animal Welfare (OLAW). Mice received apabetalone at $150 \mathrm{mg} / \mathrm{kg}$ b.i.d. or vehicle by oral gavage for 3 consecutive days. Animals were anesthetized with isoflurane and sacrificed by cardiac puncture and exsanguination. Next, whole livers were harvested, rinsed in cold PBS, flash-frozen in liquid nitrogen, and stored at $-80^{\circ} \mathrm{C}$. Total RNA was extracted from chimeric livers with TRI$\mathrm{zol}^{\circledR}$ Reagent and was reverse transcribed with High-Capacity cDNA Reverse Transcription Kit, followed by rtPCR with human TaqMan ${ }^{\mathrm{TM}}$ Gene Expression Assays and TaqMan ${ }^{\mathrm{TM}}$ Gene Expression Master Mix (ThermoFisher Scientific). 
2.7.2. Endotoxemia Mouse Model. Prior to LPS administration, eight-week-old male $\mathrm{C} 57 \mathrm{BL} / 6$ mice received vehicle [11] or apabetalone $(150 \mathrm{mg} / \mathrm{kg}$ b.i.d., formulation EA006) by gavage for 6 days (AraVasc Inc., Moffett Field, CA). On day 7 , mice received apabetalone $4 \mathrm{~h}$ prior to an intraperitoneal injection of E. coli 0111:B4 LPS (10 $\mu$ g per mouse) (Sigma-Aldrich) and again at the time of the LPS injection. Animals were sacrificed $24 \mathrm{~h}$ after the LPS injection on day 8. Liver and plasma (Li-heparin) were harvested for gene expression and protein analysis. Total RNA extracted from livers $\left(\mathrm{TRIzol}^{\circledR}\right.$ ) was reverse transcribed with High-Capacity cDNA Reverse Transcription Kit (ThermoFisher Scientific). Gene expression was measured as previously described [16] using mouse $\beta$-actin as an endogenous control. Statistical significance was determined through 1-way ANOVA followed by Tukey's multiple comparison test; $p \leq 0.05$ was considered statistically significant.

2.8. SOMAscan ${ }^{T M}$ Proteomic Analysis. The full design and rationale of the ASSERT study (NCT01058018) have been published previously [19]. Patients with stable coronary artery disease (CAD) and on statin therapy received placebo or $100 \mathrm{mg}$ apabetalone twice per day. Baseline and end-ofstudy (12 weeks) plasma samples from ASSERT were analyzed using the SOMAscan ${ }^{\mathrm{TM}} 1.3 \mathrm{~K}$ proteomic technology (SomaLogic Inc., Boulder, CO) that measures the relative abundance of 1305 proteins [20]. Shapiro-Wilk tests were used to determine data distribution. For normally distributed parameters, paired Student's $t$-tests were used to calculate statistical significance versus baseline, while for nonnormally distributed parameters, Wilcoxon signed-rank tests were applied versus baseline. Mann-Whitney $U$ test was used to compare median percent change between apabetalonetreated patients and placebo. Proteins affected by apabetalone treatment (versus placebo) by more than $10 \%(p<0.05)$ were analyzed with $\mathrm{IPA}^{\circledR}$ software using canonical pathway and upstream regulator analytics.

\section{Results}

3.1. Apabetalone Downregulates Basal Expression of APR Genes in Human Hepatocytes. Previously, we have reported that treatment with apabetalone reduced the expression of gene sets within the complement, coagulation, and APR pathways in primary human hepatocytes $[12,13,16]$. Here, we confirm the apabetalone-driven downregulation of several APR genes in nonstimulated hepatocytes obtained from human donors ( $72 \mathrm{~h}$ treatment shown in Figure 1(a)). Basal secretion of APR proteins known to correlate with CVD [21-23], including serum amyloid $\mathrm{P}$ (encoded by $A P C S$ ), ceruloplasmin (encoded by $C P$ ), and plasminogen activator inhibitor-1 (encoded by SERPINE1), was also significantly lowered by apabetalone treatment (by 26-82\%, Figure $1(\mathrm{~b})$ ). Thus, BET inhibition by apabetalone can downregulate APR gene transcription and protein abundance in noninflammatory, basal conditions in vitro.

To assess effects of apabetalone on basal levels of APR gene expression in vivo, we administered apabetalone to homozygous albumin enhancer/promoter-driven urokinase- type plasminogen activator/severe combined immunodeficient (uPA/SCID) mice with humanized liver [24]. In this chimeric liver model, replacement of mouse hepatocytes with $\mathrm{PHH}$ ranges from 70 to $90 \%$, allowing for the study of human hepatocytes in vivo. Mice were treated with $150 \mathrm{mg} / \mathrm{kg}$ b.i.d. apabetalone or vehicle followed by liver mRNA analysis. Apabetalone reduced expression of human APR genes $A 2 M$ (encoding $\alpha$-2-macroglobumin), APCS, CP, F2 (encoding thrombin), IL1RN (encoding IL-1 receptor antagonist), IL18, ORM1 (encoding orosomucoid 1), SERPINA1 (encoding $\alpha$-1 antitrypsin), SERPINE1, and CRP ( $p<0.05$, except for the latter two genes where $p \leq 0.1$ ) (Figure $1(\mathrm{c})$ ). Overall, this data demonstrates that apabetalone can downregulate APR gene transcription in nonstimulated human primary cells and in humanized mouse liver.

\subsection{Apabetalone Downregulates Cytokine-Induced APR Gene} Expression in Hepatocytes. To study hepatocyte response to BET inhibition under inflammatory conditions, we treated primary human hepatocytes with cytokines $(10 \mathrm{ng} / \mathrm{mL}$ IL-6 or IL-1 $\beta$ ) and apabetalone for $72 \mathrm{~h}$ and assessed mRNA transcription and secretion of APR proteins. CRP, SAA1/2 (encoding serum amyloid A), $C P, A 2 M, H P$ (encoding haptoglobin), APCS, and ORM1 were induced after a $72 \mathrm{~h}$ exposure to IL-6 (Figure 2(a)), while CRP, SAA1/2, and ORM1 expression also increased with IL-1 $\beta$ treatment (Figure $2(\mathrm{c})$ ). The CRP mRNA level was most sensitive to apabetalone treatment. Apabetalone reduced IL-6 mediated CRP induction by $69 \%$ at the mRNA level and $78 \%$ at the secreted protein level (Figures 2(a) and 2(b)) [16], whereas IL-1 $\beta$-mediated induction of CRP gene and protein expression was reduced by apabetalone by $77 \%$ and $70 \%$, respectively (Figures 2 (c) and 2(d)). IL-6-induced A2M and APCS expression was less sensitive to apabetalone, with $<50 \%$ reduction at the protein level (Figure 2(b)). Thus, APR gene stimulation by distinct cytokines is differentially sensitive to a BET inhibitor.

To evaluate the effect of BET inhibition on liver inflammatory responses to complex cytokine signaling in vivo, mice were pretreated with $150 \mathrm{mg} / \mathrm{kg}$ apabetalone (6 days) before systemic inflammation was induced by lipopolysaccharide (LPS) injection. As CRP gene regulation differs between mice and humans [25], expression of other model APR genes was examined in the liver post-LPS injection. Apcs, Saa1, and $A 2 \mathrm{~m}$ genes were induced $24 \mathrm{~h}$ after LPS injection, and coadministration of apabetalone suppressed Apcs and $A 2 \mathrm{~m}$ by $44 \%$ and $88 \%$, respectively (Figure $2(\mathrm{e})$ ). To assess the effect of apabetalone on innate immune response in the liver, we surveyed immune cell marker expression at 24 hours post-LPS injection (Figure 2(f)). Analysis revealed a suppressive effect of apabetalone on Cd14 mRNA (encoding an LPS receptor expressed by monocytes, neutrophils, Kupffer cells, sinusoidal endothelial cells, and hepatocytes) and on Ccr2 mRNA (encoding a chemokine receptor expressed on monocytes, Kupffer cells, and hepatic stellate cells). Apabetalone treatment did not impact the expression of macrophage and Kupffer cell markers Cd68, Aif1, and Marco (Figure 2(f)). Results demonstrate in vivo sensitivity of $\mathrm{APR}$ and innate immune genes to pharmacological inhibition of BET proteins during liver inflammation. 


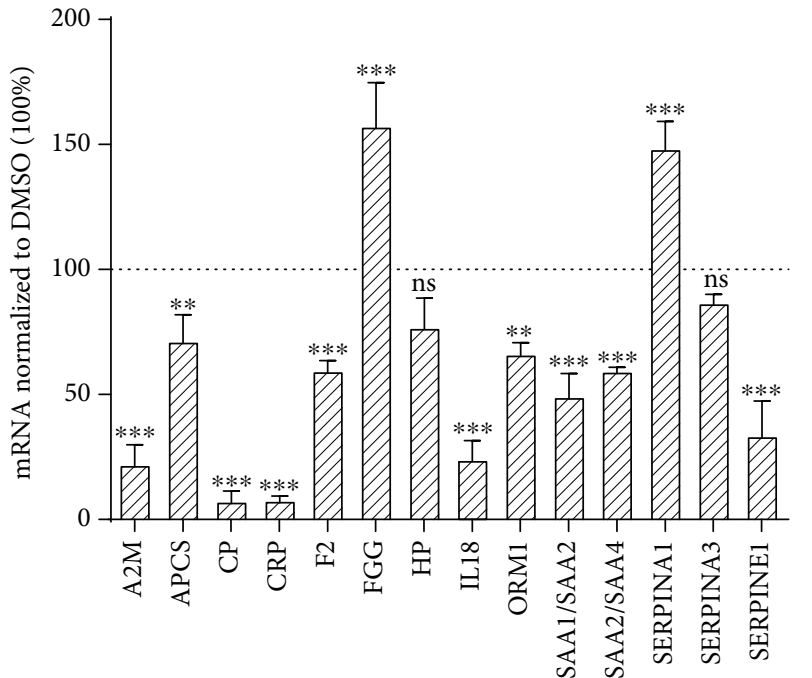

(a)

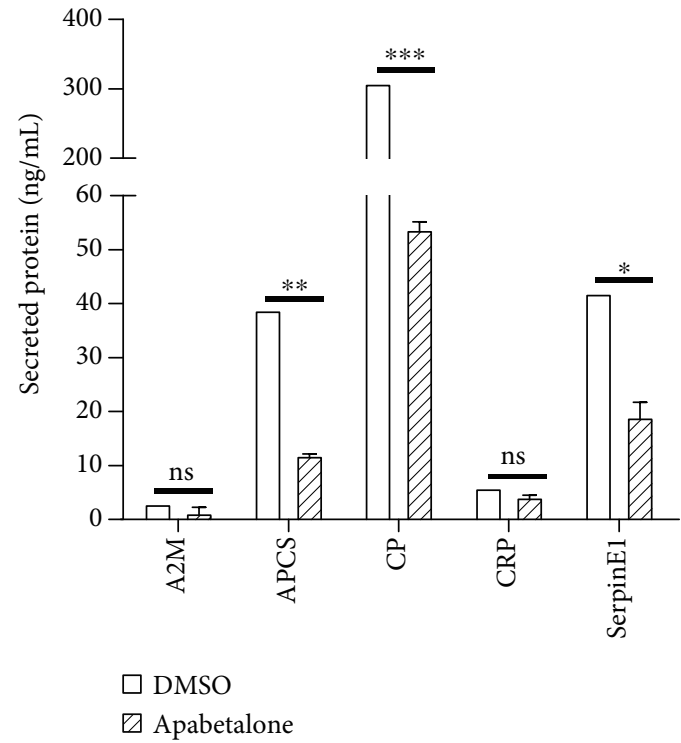

(b)

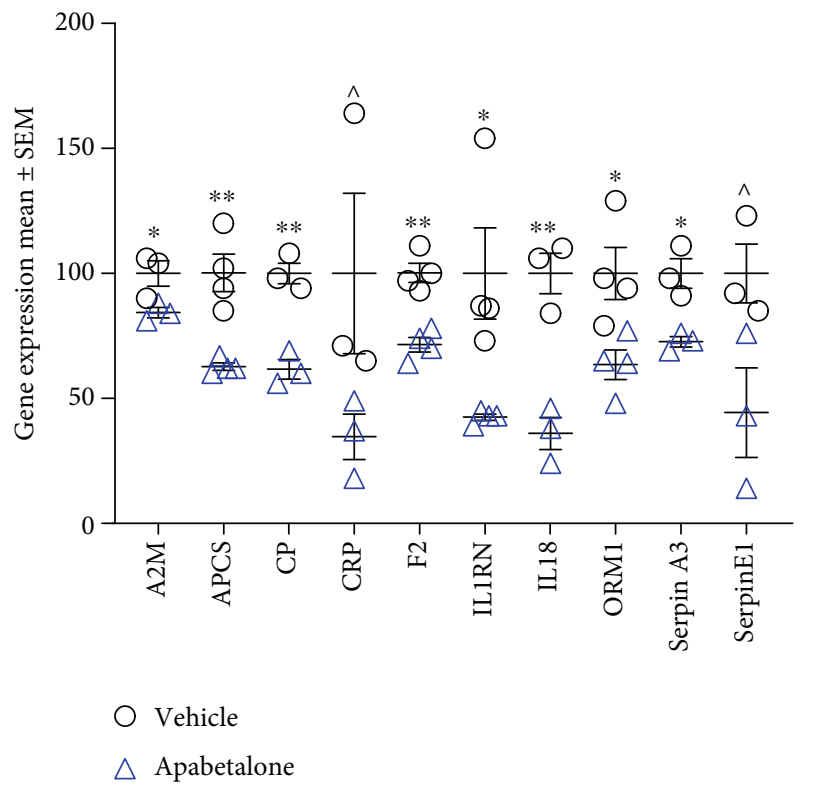

(c)

FIGURE 1: Apabetalone alters basal expression of genes within the APR pathway under noninflammatory conditions in human hepatocytes. (a) rtPCR shows that apabetalone $(30 \mu \mathrm{M})$ treatment decreases the expression of APR genes $(72 \mathrm{~h})$. Gene expression is normalized to vehicle (DMSO) treated cells. (b) Apabetalone $(30 \mu \mathrm{M})$ decreases APR protein secretion after $72 \mathrm{~h}$ of treatment. Protein secretion in the final $24 \mathrm{~h}$ of treatment is measured by ELISA and is expressed as nanograms per milliliter of tissue culture media. Representative data mean \pm S.D. is shown. (c) APR gene expression in humanized liver of chimeric mice. Mice received $150 \mathrm{mg}$ b.i.d. apabetalone or vehicle treatment for 1 to 3 days. Mean data normalized to naïve samples \pm S.E.M. is shown. Statistical significance was determined through $t$-test comparison of vehicle and apabetalone treatment, where ${ }^{*} p<0.05,{ }^{* *} p<0.01,{ }^{* * *} p<0.001$, and ns means no significant difference.

3.3. BET Proteins Mediate the Induction of CRP Gene Expression in Response to Cytokines. To examine the mechanism of CRP gene induction under inflammatory conditions, we used the HepaRG ${ }^{\mathrm{TM}}$ cell line, an established surrogate for human primary hepatocytes that offers robust responses to inflammatory stimuli without interdonor variability [26]. IL- 6 and IL- $1 \beta$ have been previously shown to modulate CRP expression in hepatocytes within a few hours of stimulation $[2,3]$. Here, short-term $(2 \mathrm{~h})$ individual cytokine treatment of $\mathrm{HepaRG}^{\mathrm{TM}}$ cells moderately increased $C R P$ expression (Figure 3(a)). Simultaneous exposure to both IL6 and IL-1 $\beta$ (dual cytokine treatment) further increased levels of the CRP mRNA (66-fold) (Figure 3(a)). Pretreatment with apabetalone, followed by a $2 \mathrm{~h}$ cotreatment with cytokines, significantly repressed dual cytokine induction of CRP mRNA by 52\% (Figure 3(a)). CRP mRNA levels were similarly, albeit less robustly, modulated in primary human hepatocytes (Figure 3(b)), confirming that HepaRG ${ }^{\mathrm{TM}}$ cells 


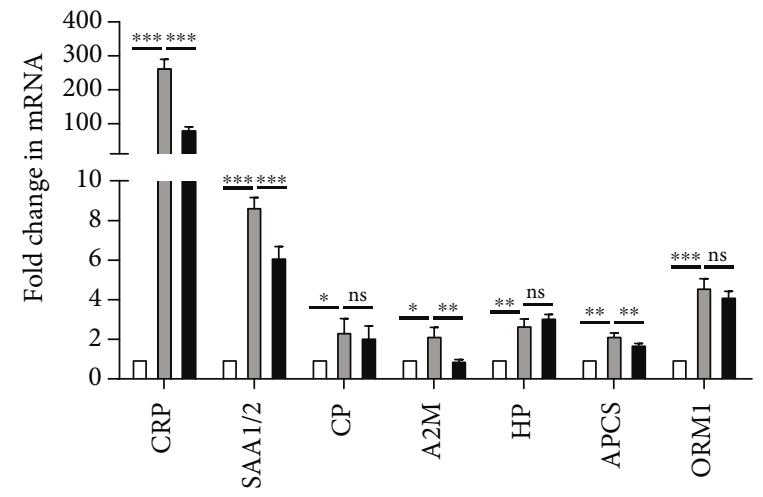

$\square$ Naive

$\square$ IL-6

- IL-6+apabetalone

(a)

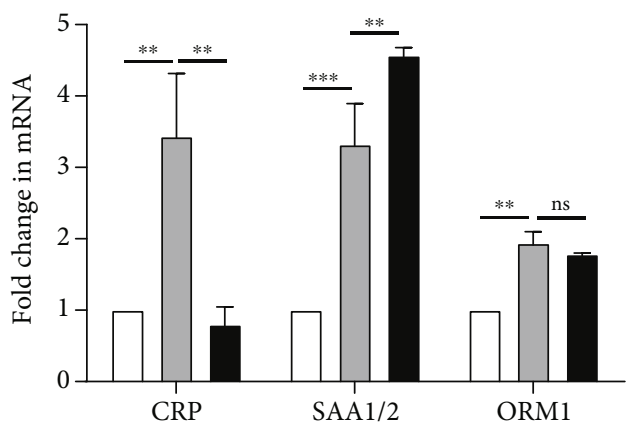

$\square$ Naive

$\square$ IL- $1 \beta$

- IL- $1 \beta+$ apabetalone

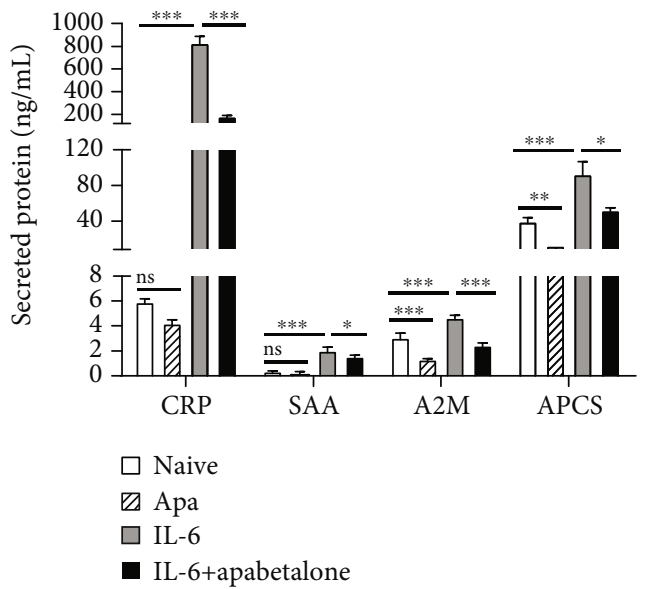

(b)

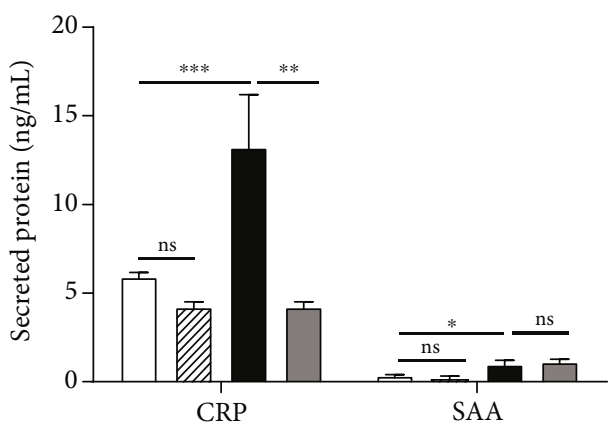

$\square$ Naive

$\square$ Apabetalone

- IL-1 $\beta$

$\square$ IL- $1 \beta$ +apabetalone

(d)

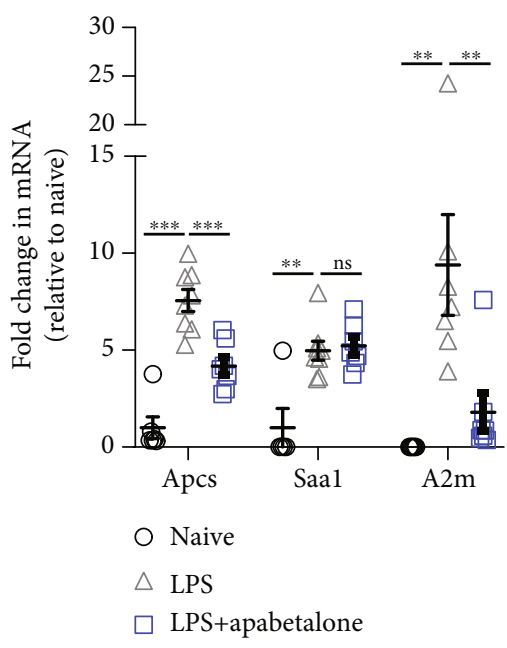

(e)

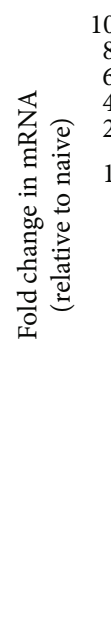

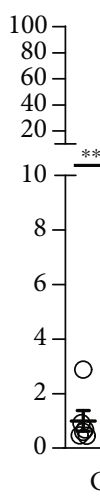

20 土******

$10 \stackrel{* * * * *}{\Delta}$

$\triangle \Delta \Delta$

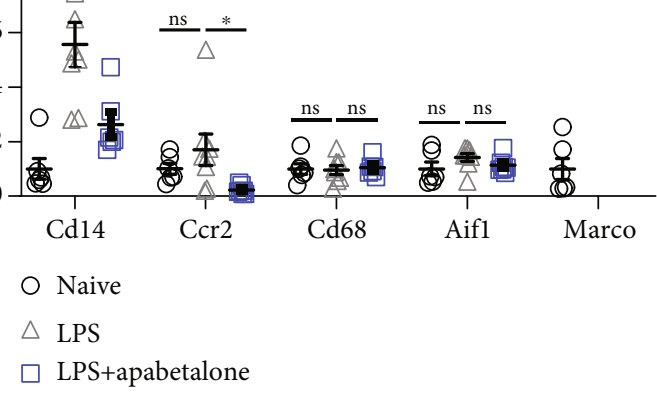

(f)

Figure 2: Apabetalone downregulates cytokine-induced expression of APR genes in hepatocytes. (a, b) Apabetalone (25 $\mu \mathrm{M})$ reduces IL-6 mediated induction ( $72 \mathrm{~h}$ ) of CRP, A2M, and APCS mRNA (rtPCR) (a) and protein (ELISA) (b). Data is presented as the mean \pm S.D. (c, d) Apabetalone $(25 \mu \mathrm{M})$ reduces IL- $1 \beta$-mediated induction of CRP mRNA (c) and protein (d). Data is presented as the mean \pm S.D. (e) Hepatic APR gene expression is downregulated by apabetalone in the LPS mouse model of systemic inflammation ( $\mathrm{rtPCR}, 24 \mathrm{~h}$ post-LPS injection). (f) Innate immune gene expression in mouse liver (real-time PCR, $24 \mathrm{~h}$ post-LPS injection). (e, f) Data acquired from 6 to 8 animals per group and presented as a mean \pm S.E.M. normalized to naïve mice. Statistical significance was determined through 1 -way ANOVA analysis followed by Tukey's multiple comparison test, where ${ }^{*} p<0.05,{ }^{* *} p<0.01,{ }^{* * *} p<0.001$, and ns means nonsignificant. 


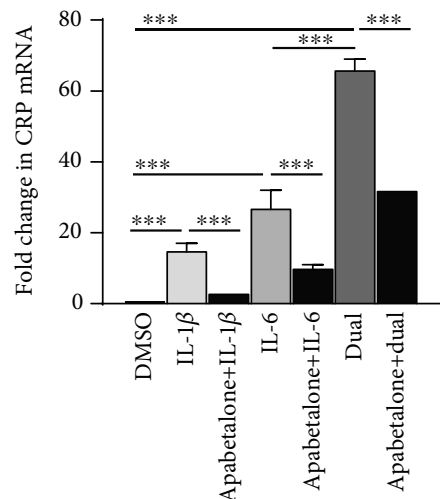

(a)

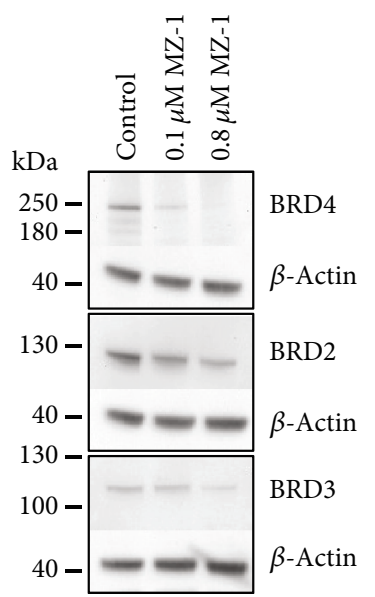

(c)

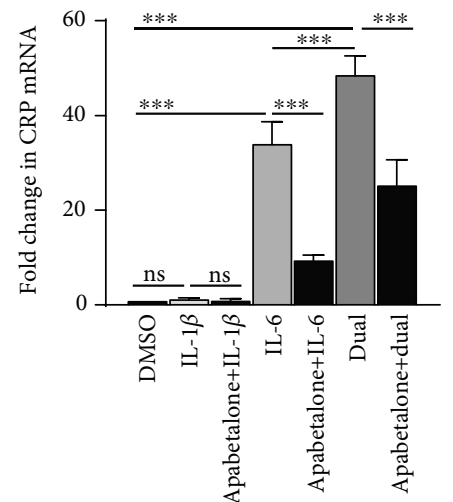

(b)

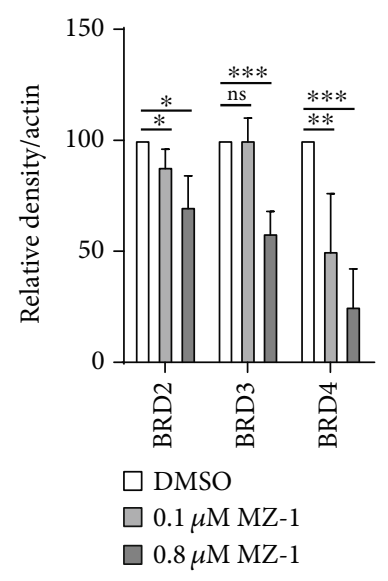

(d)
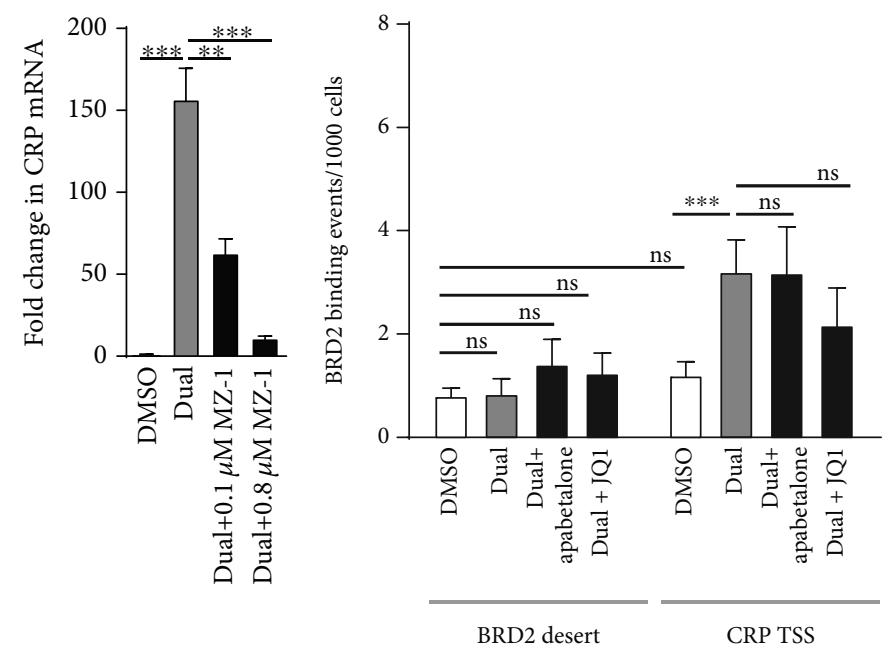

(e)

(f)

FIgURe 3: Continued. 


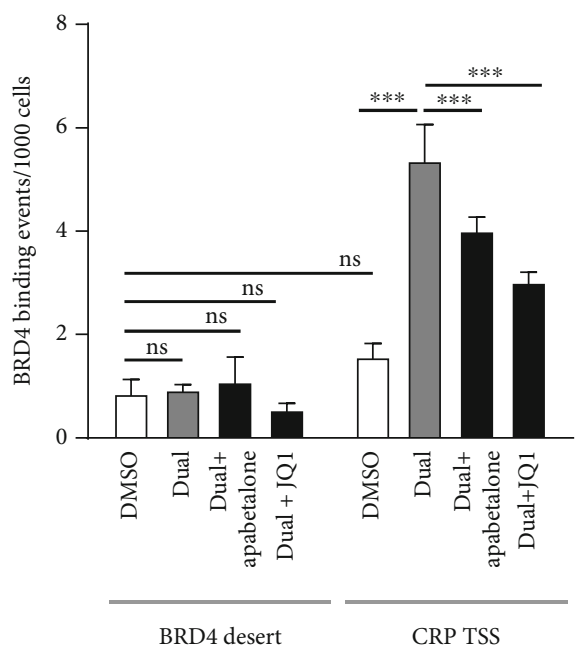

(g)

Figure 3: BET proteins contribute to cytokine-mediated induction of the CRP gene. CRP gene expression changes in $\mathrm{HepaRG}^{\mathrm{TM}}$ cells (a) and human primary hepatocytes (b) measured by rtPCR in response to IL-6, IL-1 $\beta$, or combined (dual) cytokine treatment ( $2 \mathrm{~h}$ ). Apabetalone pretreatment $(1 \mathrm{~h}, 25 \mu \mathrm{M})$ counters mRNA induction in response to single or dual cytokine treatment. Gene expression is graphed relative to DMSO-treated cells. Representative data from three independent repeats is shown. Data is presented as a mean \pm S.D. (c) MZ-1 degrades BRD2, BRD3, and BRD4 protein in HepaRG ${ }^{\mathrm{TM}}$ cells in a dose-dependent manner. Representative Western blot data is shown. (d) Quantification of BRD2, BRD3, and BRD4 protein bands relative to $\beta$-actin. Data is presented as the mean of four independent replicates \pm S.D. (e) BET degradation by MZ-1 significantly repressed dual cytokine-induced CRP transcription. Representative data from three independent repeats is shown. Data is presented as the mean \pm S.D. (f, g) Dual cytokine stimulation (2h) increases BRD2 (f) and BRD4 (g) occupancy on the CRP transcription start site (CRP TSS) but not in a BET protein-lacking region (desert) as determined by ChIP. Pretreatment $(1 \mathrm{~h})$ with apabetalone $(25 \mu \mathrm{M})$ or JQ1 $(0.5 \mu \mathrm{M})$ reduces BRD4 association with the CRP promoter (g). Samples were processed in triplicate. Data is presented as the mean \pm S.D. Statistical significance was determined through 1-way ANOVA followed by Tukey's multiple comparison test, where ${ }^{*} p<0.05,{ }^{* *} p<0.01,{ }^{* * *} p<0.001$, and ns means no significant difference.

are suitable for studying hepatocyte response to cytokine signaling. Preincubation of HepaRG ${ }^{\mathrm{TM}}$ cells with apabetalone did not interfere with immediate (0 to 30 minutes) signaling through IL-6 or Il- $1 \beta$ receptors as phosphorylation of STAT3 Tyr705 and NF- $\kappa \mathrm{B}$ p65 Ser536 was apparent within minutes of cytokine addition in the presence of apabetalone (Supplemental Figure 1). Together, this data suggests a direct involvement of BET proteins in early CRP gene transcription downstream of cytoplasmic inflammatory signaling.

To confirm that BET proteins play a role in cytokinemediated CRP gene regulation, HepaRG ${ }^{\mathrm{TM}}$ cells were exposed to the proteolysis targeting chimeric compound (PROTAC) MZ-1 that directs BET proteins for degradation by the proteasome [27]. A $24 \mathrm{~h}$ incubation with either $0.1 \mu \mathrm{M}$ or $0.8 \mu \mathrm{M}$ MZ-1 decreased BRD2 and BRD4 protein abundance in cell lysates (up to $30 \%$ or $75 \%$, respectively). BRD3 levels were not sensitive to $0.1 \mu \mathrm{M} \mathrm{MZ}-1$ but were reduced by $0.8 \mu \mathrm{M}$ MZ-1 (Figures 3(c) and 3(d)). 0.1 $\mu \mathrm{M} \mathrm{MZ-1-driven}$ $\mathrm{BRD} 2$ and BRD4 protein reduction was accompanied by a $60 \%$ decrease in dual cytokine-induced CRP gene expression (Figure 3(e)), indicating that these BET proteins contribute significantly to the cytokine-induced transcription of CRP.

To determine whether BRD2 and BRD4 proteins are directly involved in $C R P$ gene transcription, we performed chromatin immunoprecipitation assays (ChIP). Dual cytokine stimulation $(2 \mathrm{~h})$ induced a 3.2-fold enrichment of $\mathrm{BRD} 2$ on the CRP promoter, but this increase was not sensitive to competitive BD inhibition (Figure 3(f)). Cytokine treatment also evoked a significant increase of 5.3-fold in BRD4 association with the CRP promoter region (CRP TSS, Figure $3(\mathrm{~g}))$. This enrichment was countered by pretreatment with apabetalone (25\% reduction) or JQ1 (44\% reduction) indicating that it was BD-dependent (Figure 3(g)). Overall, data suggests a predominant contribution of BRD4 to the increase in $C R P$ gene transcription following cytokine stimulation of hepatocytes. BRD4 inhibitors, including apabetalone, counter the BRD4 interaction with the CRP promoter, consistent with the decrease in CRP gene transcript levels measured after treatment (Figure 3(a)).

3.4. Apabetalone Downregulates Circulating Targets of the APR Pathway and Cytokine Signaling in CAD Patients. Elevated circulating APR proteins are markers of chronic inflammation and correlate with CVD [28]. To assess the effects of BET inhibition by apabetalone on circulating inflammatory mediators, SOMAscan ${ }^{\mathrm{TM}} 1.3 \mathrm{~K}$ proteomic analysis [20] was performed on plasma samples from patients with stable $\mathrm{CAD}$ on standard-of-care therapy, treated with placebo or $100 \mathrm{mg}$ b.i.d. apabetalone for 12 weeks $(n=55$; as described in [19]). Changes in plasma protein abundance specific to apabetalone treatment $(>10 \%$ difference versus placebo, $p<0.05$ ) were analyzed using the "canonical pathway" and "upstream regulator" bioinformatics tools with the Ingenuity ${ }^{\circledR}$ Pathway Analysis (IPA $\left.{ }^{\circledR}\right)$ software. The "canonical pathway" analytic tool ranked "APR signaling" as the top pathway significantly downregulated by apabetalone in plasma from CAD patients 
TABLE 1: Apabetalone downregulates transcriptional targets of inflammatory mediators in the CAD patient plasma as predicted by IPA ${ }^{\circledR}$ "canonical pathway" and "upstream regulators" analysis.

\begin{tabular}{|c|c|c|c|c|}
\hline $\begin{array}{l}\text { Ingenuity }{ }^{\circledR} \\
\text { Pathway } \\
\text { Analysis }\end{array}$ & Pathway/regulator ${ }^{\mathrm{a}}$ & $\begin{array}{l}\text { Activation } \\
z \text {-score }\end{array}$ & $\begin{array}{l}p \text { value of } \\
\text { overlap }^{c}\end{array}$ & Target molecules in dataset (gene symbols) ${ }^{\mathrm{d}}$ \\
\hline $\begin{array}{l}\text { Canonical } \\
\text { pathway }\end{array}$ & APR signaling & -2.1 & $2.4 \times 10^{-10}$ & C4A/C4B, C3, IL1RN, CRP, MAPK9, FGA/FGB/FGG, AGT \\
\hline $\begin{array}{l}\text { Upstream } \\
\text { regulators }\end{array}$ & LPS & -3.1 & $8.9 \times 10^{-13}$ & $\begin{array}{l}\text { ADIPOQ, AGT, BCL6, C3, CCL18, CCL5, CDH1, CDH5, CRP, CSF1R, } \\
\text { EREG, FGA/FGB/FGG, IL1RN, IL2, KIT, MAPK9, MB, MMP10, NCR3, } \\
\text { POMC, SPP1, TIMP2, TNFSF14, VTN }\end{array}$ \\
\hline $\begin{array}{l}\text { Upstream } \\
\text { regulators }\end{array}$ & IL-6 & -2.6 & $7.1 \times 10^{-15}$ & $\begin{array}{l}\text { AGT, BCL6, C3, CCL5, CDH1, CDKN1B, CRP, EREG, FGA/FGB/FGG, } \\
\text { GCG, IGFBP6, IL1RN, IL2, KIT, MMP10, POMC, SPP1 }\end{array}$ \\
\hline $\begin{array}{l}\text { Upstream } \\
\text { regulators }\end{array}$ & IFNG & -2.6 & $2.5 \times 10^{-10}$ & $\begin{array}{c}\text { ADIPOQ, AGT, C3, C4A/C4B, CCL18, CCL5, CDH1, CDKN1B, CSF1R, } \\
\text { CTSD, ERAP1, FGA/FGB/FGG, IL1RN, IL2, MMP10, NCR3, POMC, } \\
\text { SPP1, TNFSF12 }\end{array}$ \\
\hline $\begin{array}{l}\text { Upstream } \\
\text { regulators }\end{array}$ & OSM & -2.0 & $6.5 \times 10^{-7}$ & $\begin{array}{l}\text { CCL5, SPP1, MMP10, IGFBP6, FGA/FGB/FGG, CDKN1B, } \\
\text { CDH1, CRP, POMC }\end{array}$ \\
\hline $\begin{array}{l}\text { Upstream } \\
\text { regulators }\end{array}$ & IL1A & -2.0 & $2.0 \times 10^{-6}$ & CCL5, IL1RN, IL2, KIT, MMP10, POMC, SPP1 \\
\hline $\begin{array}{l}\text { Upstream } \\
\text { regulators }\end{array}$ & NFKB1 & -1.8 & $5.2 \times 10^{-5}$ & CCL5, CRP, FGA/FGB/FGG, IL1RN, IL2, MMP10 \\
\hline
\end{tabular}

Plasma proteins affected by apabetalone treatment by more than $10 \%$ (versus placebo, $p<0.05$ ) were analyzed with the IPA ${ }^{\circledR}$ "canonical pathway" and "upstream regulator" analytics. ${ }^{a}$ APR: acute phase response; LPS: lipopolysaccharide; IL-6: interleukin 6; IFNG: interferon $\gamma$; OSM: oncostatin M; IL1A: interleukin $1 \alpha$; NFKB1: nuclear factor $\kappa$ B subunit $1 .{ }^{b}{ }^{b} A^{\circledR} z$-score compares the observed differential regulation of a gene in the dataset to changes predicted by the literature which can be either "activating" or "inhibiting." $z-$ score $<-2$ predicts downregulation within a gene set associated with a transcriptional regulator. ${ }^{c}$ The overlap $p$ value measures whether there is a statistically significant overlap between the dataset genes and the genes that are regulated by a transcriptional regulator. It is calculated using Fisher's exact test, and significance is attributed to $p$ values $<0.01$. ${ }^{\mathrm{d}}$ Apabetalone target proteins that contribute to IPA ${ }^{\otimes}$ "canonical pathways" or "upstream regulators" are listed as gene symbols.

(versus placebo, $p$ value $=2.4 \times 10^{-10}, z$-score $=-2.1$ ) (Table 1). The IPA ${ }^{\circledR}$ “upstream regulator” analysis was used to group plasma proteins affected by apabetalone treatment as transcriptional targets of specific upstream activators. This analysis highlighted downstream signaling by LPS, IL-6, interferon $\gamma($ IFN $\gamma)$, IL- $1 \alpha$, the IL-6-like cytokine oncostatin $\mathrm{M}$ (OSM), and the nuclear factor $\kappa \mathrm{B}$ subunit 1 (NFKB1), as significantly suppressed by apabetalone versus placebo (predicted downregulation with a $z-$ score $<-2$, $p$ value $<0.05$ ) (Table 1). No significant changes were detected in plasma levels of IL- 6 , interferon $\gamma$ (IFN $\gamma$ ), and IL- $1 \beta$ between placeboand apabetalone-treated CAD patients (not shown). However, as indicated by the IPA ${ }^{\circledR}$ "upstream regulator" analysis, a number of circulating plasma proteins regulated by these cytokines, including CRP, IL-1R antagonist, and fibrinogen $\gamma$, were reduced by apabetalone treatment (Figure 4). These are liverderived APR proteins, indicating that apabetalone impacts APR protein secretion from hepatocytes in humans. Taken together, the plasma proteomics data from CAD patients demonstrates the modulatory effect of apabetalone on circulating APR proteins and inflammatory pathways linked to progression of CVD.

\section{Discussion}

Systemic inflammatory signaling that accompanies chronic disease leads to altered production of APR proteins by the liver [1]. Changes in the transcriptome and the proteome associated with cytokine signaling in hepatocytes have been extensively investigated, but mechanisms that control APR gene transcription are not fully understood. This is due to the complexity of hepatocyte responses to cytokines, glucocorticoids, and growth factors secreted during APR that lead to activation of multiple parallel pathways $[2,29]$. Transcription factors previously implicated in the regulation of APR expression differ substantially between genes and show dynamic time- and stimulus-dependent responses [2, 29]. Members of the STAT, NF- $\kappa \mathrm{B}, \mathrm{C} / \mathrm{EBP}$, hepatocyte nuclear factor, AP-1, and Smad protein families have been detected on APR gene promoters, including CRP, in hepatocarcinoma cells and in primary hepatocytes [2, 29-32] (and references therein). Here, we identify BET proteins as epigenetic readers that regulate $\mathrm{APR}$ gene transcription. We use the BET inhibitor apabetalone, developed to treat CVD, as a tool compound to counter the activity of BET proteins in noninflammatory and inflammatory conditions in hepatocytes in vitro, in mice and in CAD patients.

The best characterized BET protein, BRD4, plays a crucial role in inflammatory responses $[7,8]$. BRD4 occupies transcriptionally active chromatin regions through its interactions with $\mathrm{H} 3 \mathrm{~K} 27 \mathrm{ac}[29,30,33]$. In mouse hepatocytes, IL- $1 \beta$ and IL- 6 dual cytokine signaling has been shown to increase the abundance of H3K27ac, STAT3, NF- $\kappa \mathrm{B}$, and RNA polymerase II in the vicinity of induced APR genes [3]. BRD4 cooperates with both STAT3 and NF- $\kappa$ B to transactivate RNAPII in nonhepatocytes [31, 32, 34]; thus, it is conceivable that it plays a similar role in activated hepatocytes. Here, we provide evidence that BRD4 is a novel 


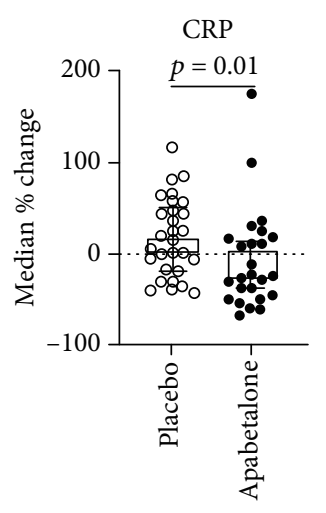

(a)

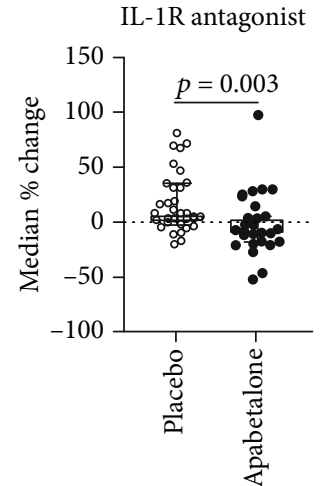

(b)

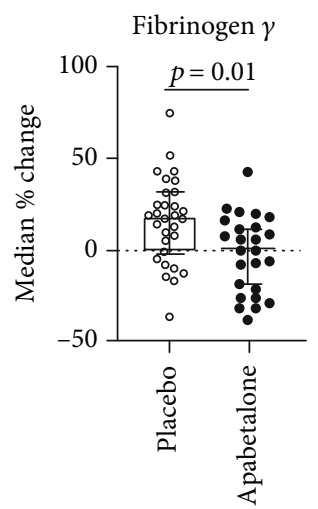

(c)

FIGURE 4: Apabetalone treatment reduces the abundance of liver-derived APR plasma proteins in CAD patients versus placebo. (a-c) Boxes represent median values of percent changes between start and end of study in each patient. Lines represent the $95 \%$ confidence interval.

transcriptional regulator required for $C R P$ expression in stimulated hepatocytes. Degradation of BET proteins by the proteolysis-inducing compound MZ-1 potently suppresses $C R P$ gene induction by IL- 6 and IL- $1 \beta$, reinforcing the notion that BET proteins are required for stimulation-dependent transcription. This is likely a direct effect, as BRD2 and $\mathrm{BRD} 4$ recruitment to the CRP promoter is detected early on during dual cytokine stimulation $(2 \mathrm{~h})$, and a short-term treatment with apabetalone or JQ1 (a pan-BETi with a different scaffold) counters the CRP promoter occupancy by BRD4 and the CRP gene induction by cytokines. Further investigation will determine whether BRD2 and BRD4 cooperate with other transcription factors to promote hepatic CRP transcription during inflammation.

We also show that long-term BET inhibition with apabetalone treatment (up to $72 \mathrm{~h}$ ) downregulates basal levels of several APR genes in human hepatocytes. This coordinated sensitivity of APR gene expression to apabetalone indicates that BET proteins are involved (directly or indirectly) in regulating this key hepatic function. Of note, suppression of APR genes by apabetalone was consistent across hepatocyte models, including in vitro-cultured human primary cells and chimeric mice with humanized livers. Moreover, mRNA and protein expression of APR proteins linked to CVD, including serum amyloid $\mathrm{P}$, ceruloplasmin, and plasminogen activator inhibitor-1 [21-23], was also lowered by apabeta- lone treatment. Although serum amyloid $\mathrm{P}$ and plasminogen activator inhibitor- 1 were not reduced in stable CAD patients analyzed here, their downregulation by apabetalone was previously observed in patients with advanced CAD [16] and in chronic kidney disease patients [17], respectively. Thus, disease type and severity may influence the sensitivity of the APR proteins to apabetalone.

During endotoxemia, apabetalone suppressed hepatocyte response to LPS, as shown by downregulation of mouse model APR genes APCS and A2M. This in vivo effect likely results from direct modulation of gene transcription by apabetalone as demonstrated in in vitro experiments with stimulated hepatocytes. However, apabetalone may also act indirectly by impacting infiltrating or liver-resident innate immune cells, which stimulate hepatocytes to produce APR proteins during endotoxemia. Innate immune marker profiling showed that apabetalone suppressed the inflammatory expression of the Cd14 gene, which encodes an LPS coreceptor displayed on monocytes, neutrophils, Kupffer cells, sinusoidal endothelial cells, and hepatocytes $[35,36]$. As previously reported [37], Cd14 expression level is relatively low in normal liver but sharply increases following LPS treatment (also shown in Figure 2(f)), facilitating LPS uptake by cells. Downregulation of $C d 14$ mRNA by BETi treatment would potentially reduce LPS signaling in the liver, attenuating the hepatocyte inflammatory response $[38,39]$. Apabetalone also 
reduced the mRNA level of $C c r 2$, a key chemokine receptor mainly found on the surface of infiltrating monocytes, macrophages, and resident Kupffer cells [40]. Ccr2 functions to recruit these cells to the site of inflammation, and its reduction by apabetalone may attenuate immune cell activity in the liver. Benefits of BETi treatment for inflammatory liver disease are currently being investigated [41, 42].

Enhanced inflammatory signaling accompanies several chronic human diseases including CVD, where elevated plasma APR proteins are being used as biomarkers of systemic inflammation [35]. Stable CAD patients studied here had elevated hsCRP levels $(3.9 \pm 0.3 \mathrm{mg} / \mathrm{L})$ despite standard-ofcare therapy [16], reflective of elevated systemic cytokine signaling. To study the effect of apabetalone in CAD patients, we examined changes in $1300+$ plasma proteins in response to drug treatment over the course of 3 months, versus placebo. Bioinformatics analysis of the plasma proteome pre- and posttreatment showed that apabetalone significantly modulates levels of multiple components of the canonical "APR signaling pathway," resulting in a predicted inhibition of the APR pathway in treated patients (Table 1). APR proteins most significantly downregulated by apabetalone were the IL-1R antagonist $(-13.8 \%, p=0.003)$, fibrinogen $\gamma$ chain $(-16.9 \%$, $p=0.01)$, and CRP $(-42.3 \%, p=0.01)$ (Figure 4), each of which positively correlates with an increased risk of CVD $[18,36]$. Apabetalone-mediated reductions in these liverderived plasma APR proteins support observations from both cellular and animal studies (this report and [12]). Further, bioinformatics analysis of the plasma proteome showed that circulating APR proteins affected by apabetalone are transcriptional targets of inflammatory mediators IL-6, IFN $\gamma$, IL- $1 \alpha$, oncostatin M (OSM), LPS, and NF- $\kappa$ B (Table 1). Consistent with this finding, several other cytokine targets were also downregulated by apabetalone in patients' plasma (Table 1) including stromelysin-2/MMP10 $(-29 \%, p<0.008)$, RANTES/CCL5 $(-29 \%, p<0.04)$, TWEAK/TNFSF12 $(-21 \%$, $p<0.002)$, osteopontin/SPP1 $(-16 \%, p<0.03)$, epiregulin/EREG $(-13 \%, p<0.01)$, PARC/CCL18 $(-13 \%, p<0.03)$, and LIGHT/TNFSF14 $(-11 \%, p<0.004)$. These proteins are linked to atherosclerotic plaque development and rupture, and their circulating levels correlate with CVD risk [18, 36-42]. Thus, downregulation of cytokine pathways that lead to APR protein expression may reduce CVD risk in apabetalonetreated patients $[43,44]$.

\section{Conclusions}

BET inhibition by apabetalone counters cytokine signaling in hepatocytes, leading to reduced APR gene expression and protein secretion, including CRP. Reduction of the CRP gene expression by apabetalone during early cytokine signaling is at least partly mediated by BRD4 removal from the CRP promoter. Apabetalone treatment downregulates circulating cytokine targets, including the APR pathway components, in stable CAD patients on standard-of-care therapy, potentially dampening chronic inflammatory signaling in a disease. Apabetalone's impact on inflammatory mediators could contribute to a reduction in CVD risk and disease outcomes $[43,44]$.

\section{Abbreviations}

A2M:

$\alpha$-2-Macroglobulin

AP-1:

Activator protein 1

APCS:

Amyloid P component, serum

APR:

BD:

Acute phase response

BET:

BETi:

BRD:

CAD:

C/EBP:

ChIP:

CKD:

CP:

CRP:

CVD:

Bromodomain

Bromodomain and extraterminal

BET inhibitor

Bromodomain and extraterminal

Coronary artery disease

H3K27:

HNF:

HP:

HsCRP:

IL:

IL-1RA: Interleukin 1 receptor antagonist

IPA ${ }^{\circledR} \quad$ Ingenuity ${ }^{\circledR}$ Pathway Analysis

LPS:

MACE:

NF- $\kappa$ B:

ORM1:

OSM:

PCR:

Lipopolysaccharide

Major acute cardiac event

Nuclear factor $\kappa \mathrm{B}$

Orosomucoid 1

Oncostatin M

PHH:

Polymerase chain reaction

P-TEFb: Positive transcription elongation factor $b$

PROTAC: Proteolysis targeting chimera

RNAPII: RNA polymerase II

rtPCR: Real-time PCR

SAA: $\quad$ Serum amyloid A

SAP: $\quad$ Serum amyloid $P$

STAT3: $\quad$ Signal transducer and activator of transcription 3

TNF: $\quad$ Tumor necrosis factor.

\section{Data Availability}

The datasets generated during and/or analyzed during the current study are available in this manuscript.

\section{Ethical Approval}

All procedures followed were in accordance with the ethical standards of the responsible committee on human experimentation (institutional and national) and with the Helsinki Declaration of 1975, as revised in 2000. Animal studies were performed at Aravasc Inc. (Sunnyvale, CA) following NIH guidelines and NASA Animal Care and Use Committee (IACUC) policy with approved protocol ARA-16-001-Y1.

\section{Consent}

Informed consent was obtained from all patients for being included in the study. 


\section{Conflicts of Interest}

All authors are employees and shareholders of Resverlogix Corp.

\section{Authors' Contributions}

S.W., D.G., and E.K. designed the experiments. S.W. and D.G. performed the experiments, analyzed the data, and interpreted the results. R.J., S.W., D.G., and E.K. designed the mouse study. E.D. analyzed the mouse tissues. C.H., R.J., M.S., J.J., and N.C.W.W. participated in the clinical trial data analysis and interpretation. S.W., D.G., L.M.T., L.F., C.H., B.D.R., and E.K. analyzed the plasma proteomics data. S.W. and S.C.S. wrote the manuscript. All authors read and approved the final manuscript.

\section{Acknowledgments}

We would like to thank Resverlogix's lab members for the intellectual input and manuscript review. This work was supported by Resverlogix Corp.

\section{Supplementary Materials}

Supplemental Figure 1: apabetalone does not affect early signaling steps by IL-6 and IL-1 $\beta$. (Supplementary Materials)

\section{References}

[1] J. Jura and A. Koj, "Regulatory Mechanisms Controlling Inflammation and Synthesis of Acute Phase Proteins," in Acute Phase Proteins - Regulation and Functions of Acute Phase Proteins, IntechOpen, 2011.

[2] J. G. Bode, U. Albrecht, D. Haussinger, P. C. Heinrich, and F. Schaper, "Hepatic acute phase proteins - regulation by IL6- and IL-1-type cytokines involving STAT3 and its crosstalk with NF- $\kappa \mathrm{B}$-dependent signaling," European Journal of Cell Biology, vol. 91, no. 6-7, pp. 496-505, 2012.

[3] I. Goldstein, V. Paakinaho, S. Baek, M. H. Sung, and G. L. Hager, "Synergistic gene expression during the acute phase response is characterized by transcription factor assisted loading," Nature Communications, vol. 8, no. 1, p. 1849, 2017.

[4] P. Filippakopoulos and S. Knapp, "Targeting bromodomains: epigenetic readers of lysine acetylation," Nature Reviews. Drug Discovery, vol. 13, no. 5, pp. 337-356, 2014.

[5] M. K. Jang, K. Mochizuki, M. Zhou, H. S. Jeong, J. N. Brady, and K. Ozato, "The bromodomain protein Brd4 is a positive regulatory component of $\mathrm{P}-\mathrm{TEFb}$ and stimulates RNA polymerase II-dependent transcription," Molecular Cell, vol. 19, no. 4, pp. 523-534, 2005.

[6] Z. Yang, J. H. N. Yik, R. Chen et al., "Recruitment of P-TEFb for stimulation of transcriptional elongation by the bromodomain protein Brd4," Molecular Cell, vol. 19, no. 4, pp. 535-545, 2005.

[7] D. F. Tough and R. K. Prinjha, "Immune disease-associated variants in gene enhancers point to BET epigenetic mechanisms for therapeutic intervention," Epigenomics, vol. 9, no. 4, pp. 573-584, 2017.

[8] T. Fujisawa and P. Filippakopoulos, "Functions of bromodomain-containing proteins and their roles in homeo- stasis and cancer," Nature Reviews Molecular Cell Biology, vol. 18, no. 4, pp. 246-262, 2017.

[9] K. G. McLure, E. M. Gesner, L. Tsujikawa et al., "RVX-208, an inducer of ApoA-I in humans, is a BET bromodomain antagonist," PLoS One, vol. 8, no. 12, article e83190, 2013.

[10] S. Picaud, C. Wells, I. Felletar et al., "RVX-208, an inhibitor of BET transcriptional regulators with selectivity for the second bromodomain," Proceedings of the National Academy of Sciences of the United States of America, vol. 110, no. 49, pp. 19754-19759, 2013.

[11] R. Jahagirdar, H. Zhang, S. Azhar et al., "A novel BET bromodomain inhibitor, RVX-208, shows reduction of atherosclerosis in hyperlipidemic ApoE deficient mice," Atherosclerosis, vol. 236, no. 1, pp. 91-100, 2014.

[12] D. Gilham, S. Wasiak, L. M. Tsujikawa et al., "RVX-208, a BET-inhibitor for treating atherosclerotic cardiovascular disease, raises ApoA-I/HDL and represses pathways that contribute to cardiovascular disease," Atherosclerosis, vol. 247, pp. 4857, 2016.

[13] S. Wasiak, D. Gilham, L. M. Tsujikawa et al., "Data on gene and protein expression changes induced by apabetalone (RVX-208) in ex vivo treated human whole blood and primary hepatocytes," Data in Brief, vol. 8, pp. 1280-1288, 2016.

[14] L. M. Tsujikawa, L. Fu, S. Das et al., "Apabetalone (RVX-208) reduces vascular inflammation in vitro and in CVD patients by a BET-dependent epigenetic mechanism," Clinical Epigenetics, vol. 11, no. 1, p. 102, 2019.

[15] D. Gilham, L. M. Tsujikawa, C. D. Sarsons et al., "Apabetalone downregulates factors and pathways associated with vascular calcification," Atherosclerosis, vol. 280, pp. 75-84, 2019.

[16] S. Wasiak, D. Gilham, L. M. Tsujikawa et al., "Downregulation of the complement cascade in vitro, in mice and in patients with cardiovascular disease by the BET protein inhibitor apabetalone (RVX-208)," Journal of Cardiovascular Translational Research, vol. 10, no. 4, pp. 337-347, 2017.

[17] S. Wasiak, L. M. Tsujikawa, C. Halliday et al., "Benefit of apabetalone on plasma proteins in renal disease," Kidney International Reports, vol. 3, no. 3, pp. 711-721, 2018.

[18] The Emerging Risk Factors Collaboration, "C-reactive protein, fibrinogen, and cardiovascular disease prediction," The New England Journal of Medicine, vol. 367, no. 14, pp. 1310-1320, 2012.

[19] S. J. Nicholls, A. Gordon, J. Johansson et al., "Efficacy and safety of a novel oral inducer of apolipoprotein a-I synthesis in statin-treated patients with stable coronary artery disease a randomized controlled trial," Journal of the American College of Cardiology, vol. 57, no. 9, pp. 1111-1119, 2011.

[20] L. Gold, D. Ayers, J. Bertino et al., "Aptamer-based multiplexed proteomic technology for biomarker discovery," PLoS One, vol. 5, no. 12, article e15004, 2010.

[21] R. T. Dadu, R. Dodge, V. Nambi et al., "Ceruloplasmin and heart failure in the Atherosclerosis Risk in Communities study," Circulation. Heart Failure, vol. 6, no. 5, pp. 936-943, 2013.

[22] R. G. Jung, T. Simard, A. Labinaz et al., "Role of plasminogen activator inhibitor-1 in coronary pathophysiology," Thrombosis Research, vol. 164, pp. 54-62, 2018.

[23] N. S. Jenny, A. M. Arnold, L. H. Kuller, R. P. Tracy, and B. M. Psaty, "Serum amyloid P and cardiovascular disease in older men and women," Arteriosclerosis, Thrombosis, and Vascular Biology, vol. 27, no. 2, pp. 352-358, 2007. 
[24] C. Tateno, Y. Yoshizane, N. Saito et al., "Near completely humanized liver in mice shows human-type metabolic responses to drugs," The American Journal of Pathology, vol. 165, no. 3, pp. 901-912, 2004.

[25] N. O. Ku and R. F. Mortensen, "The mouse C-reactive protein (CRP) gene is expressed in response to IL-1 but not IL-6," Cytokine, vol. 5, no. 4, pp. 319-326, 1993.

[26] M. Klein, M. Thomas, U. Hofmann, D. Seehofer, G. Damm, and U. M. Zanger, "A systematic comparison of the impact of inflammatory signaling on absorption, distribution, metabolism, and excretion gene expression and activity in primary human hepatocytes and HepaRG cells," Drug Metabolism and Disposition, vol. 43, no. 2, pp. 273-283, 2014.

[27] M. Zengerle, K. H. Chan, and A. Ciulli, "Selective small molecule induced degradation of the BET bromodomain protein BRD4," ACS Chemical Biology, vol. 10, no. 8, pp. 1770-1777, 2015.

[28] J. Moriya, "Critical roles of inflammation in atherosclerosis," Journal of Cardiology, vol. 73, no. 1, pp. 22-27, 2019.

[29] W. Zhang, C. Prakash, C. Sum, Y. Gong, Y. Li, J. J. Kwok et al., "Bromodomain-containing protein 4 (BRD4) regulates RNA polymerase II serine 2 phosphorylation in human CD4+ T cells," The Journal of Biological Chemistry, vol. 287, no. 51, pp. 43137-43155, 2012.

[30] J. E. Lee, Y. K. Park, S. Park et al., "Brd4 binds to active enhancers to control cell identity gene induction in adipogenesis and myogenesis," Nature Communications, vol. 8, no. 1, p. 2217, 2017.

[31] B. Huang, X. D. Yang, M. M. Zhou, K. Ozato, and L. F. Chen, "Brd4 coactivates transcriptional activation of NF-kappaB via specific binding to acetylated RelA," Molecular and Cellular Biology, vol. 29, no. 5, pp. 1375-1387, 2009.

[32] S. Ray, Y. Zhao, M. Jamaluddin, C. B. Edeh, C. Lee, and A. R. Brasier, "Inducible STAT3 NH2 terminal monoubiquitination promotes BRD4 complex formation to regulate apoptosis," Cellular Signalling, vol. 26, no. 7, pp. 1445-1455, 2014.

[33] J. D. Brown, C. Y. Lin, Q. Duan et al., "NF- $\kappa$ B directs dynamic super enhancer formation in inflammation and atherogenesis," Molecular Cell, vol. 56, no. 2, pp. 219-231, 2014.

[34] Z. Zou, B. Huang, X. Wu et al., "Brd4 maintains constitutively active NF- $\kappa \mathrm{B}$ in cancer cells by binding to acetylated RelA," Oncogene, vol. 33, no. 18, pp. 2395-2404, 2014.

[35] G. R. Geovanini and P. Libby, "Atherosclerosis and inflammation: overview and updates," Clinical Science (London, England), vol. 132, no. 12, pp. 1243-1252, 2018.

[36] C. Herder, T. de las Heras Gala, M. Carstensen-Kirberg et al., "Circulating levels of interleukin 1-receptor antagonist and risk of cardiovascular disease," Arteriosclerosis, Thrombosis, and Vascular Biology, vol. 37, no. 6, pp. 1222-1227, 2017.

[37] J. Orbe, I. Montero, J. A. Rodriguez, O. Beloqui, C. Roncal, and J. A. Paramo, "Independent association of matrix metalloproteinase-10, cardiovascular risk factors and subclinical atherosclerosis," Journal of Thrombosis and Haemostasis, vol. 5, no. 1, pp. 91-97, 2007.

[38] S. C. A. de Jager, B. W. C. Bongaerts, M. Weber et al., "Chemokines CCL3/MIP1 $\alpha$, CCL5/RANTES and CCL18/PARC are independent risk predictors of short-term mortality in patients with acute coronary syndromes," PLoS One, vol. 7, no. 9, article e45804, 2012.
[39] L. M. Blanco-Colio, "TWEAK/Fn14 Axis: a promising target for the treatment of cardiovascular diseases," Frontiers in Immunology, vol. 5, 2014.

[40] F. Carbone, F. Rigamonti, F. Burger et al., "Serum levels of osteopontin predict major adverse cardiovascular events in patients with severe carotid artery stenosis," International Journal of Cardiology, vol. 255, pp. 195-199, 2018.

[41] M. Takahashi, K.'. Hayashi, K. Yoshida et al., "Epiregulin as a major autocrine/paracrine factor released from ERK- and p38MAPK-activated vascular smooth muscle cells," Circulation, vol. 108, no. 20, pp. 2524-2529, 2003.

[42] C. P. Dahl, L. Gullestad, B. Fevang et al., "Increased expression of LIGHT/TNFSF14 and its receptors in experimental and clinical heart failure," European Journal of Heart Failure, vol. 10, no. 4, pp. 352-359, 2008.

[43] S. J. Nicholls, K. K. Ray, J. O. Johansson et al., "Selective BET protein inhibition with apabetalone and cardiovascular events: a pooled analysis of trials in patients with coronary artery disease," American Journal of Cardiovascular Drugs, vol. 18, no. 2, pp. 109-115, 2018.

[44] K. K. Ray, S. J. Nicholls, K. A. Buhr et al., "Effect of apabetalone added to standard therapy on major adverse cardiovascular events in patients with recent acute coronary syndrome and type 2 diabetes," JAMA, vol. 323, no. 16, p. 1565, 2020. 